Reimar Banis gerichteten Medizin werden Durchblutungsstörungen meist als ein rein mechanisches Problem gesehen. Beispielsweise werden venös bedingte Beingeschwüre als Abflussbehinderung gedeutet und entsprechend behandelt, etwa mit Kompressionsstrümpfen. Bei arteriellen Durchblutungsstörungen wie dem sogenannten Raucherbein (periphere arterielle Verschlusskrankheit (pAVK)) wird eine fortgeschrittene Verkalkung der Gefässinnenwände als ursächlich angesehen, die man entsprechend therapeutisch angeht. Kaum jemand unter den konventionell denkenden Medizinerkollegen käme auf die Idee, dass es bei solchen Krankheitsbildern therapeutische Alternativen gibt, die ganz anders ansetzen.

Einige bemerkenswerte Einzelfälle aus meiner naturheilkundlich ausgerichteten Praxis zeigen, dass eine seelische und feinstoffliche energetische Behandlung selbst bei therapieresistenten Krankheitsbildern verblüffende Erfolge zeigt. Die erzielten Resultate sprechen meines Erachtens gegen ein rein mechanisches medizinisches Erklärungsmodell und plädieren für eine Erweiterung des therapeutischen Horizonts. Sie zeigen, dass vordergründig körperlich-mechanische Krankheiten einen seelischen und feinstofflich-energetischen Hintergrund haben, der diese Krankheiten offenbar entscheidend mit unterhält. Im Folgenden möchte ich meine Vorgehensweise darstellen, die mit einer ganzheitlichen, Körper und Seele behandelnden Methode arbeitet, bei der seelische Konflikte im Mittelpunkt stehen.

\title{
Durchblutungsstörungen erfolgreich naturheilkundlich behandeln
}

\author{
Zur Psychoenergie scheinbar rein körperlicher Krankheiten
}

\section{Energieblockaden mit der Psychosomatischen Energetik aufdecken und auflösen}

Als Erstes möchte ich die von mir praktizierte naturheilkundliche Methode kurz vorstellen, mit der ich seit mehr als einem Jahrzehnt arbeite [1]. Mit einer Mitte der Neunzigerjahre von mir entwickelten Methode namens Psychosomatische Energetik (PSE) werden zuerst die vier Ebenen des feinstofflichen Energiesystems (vital, emotional, mental, kausal) kinesiologisch mit einem speziellen Testgerät quantitativ getestet (Abb. 1). Bei den meisten chronisch Kranken findet man einen erheblichen Energiemangel. Gelingt es, diesen Energiemangel zu beseitigen, kommt es zur Ingangsetzung körpereigener Selbstheilungsprozesse.

Die Ursachen des Energiemangels können in den meisten Fällen auf unbewusste seelische Konflikte zurückgeführt werden. Zum Testen der Konflikte werden 40 Komplexmittel bestimmter homöopathischer Hochpotenzen verwendet, die später auch therapeutisch zum Auflösen der Kon-

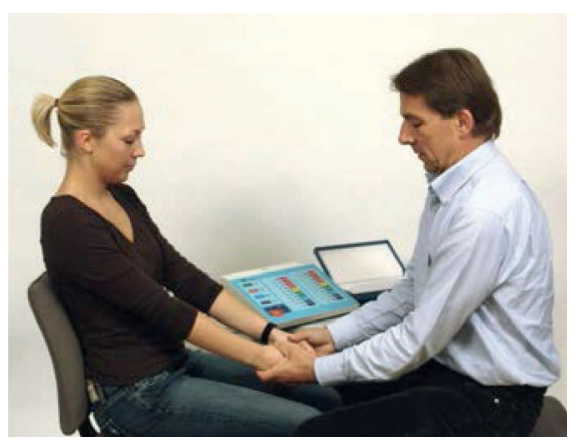

Abb. 1. Kinesiologischer Armlängentest mit Reba $^{\circledR}$-Testgerät zur Testung der Ladung der vier Energieebenen. flikte benutzt werden. Parallel mit dem Auflösen der Konflikte steigt die Energieladung der Energieebenen. Der Heilprozess läuft dabei meist sanft und häufig unbemerkt ab, wobei am häufigsten über heftige nächtliche Träume berichtet wird. Durch die PSE werden körperliche und seelische Selbstheilprozesse angeregt, wodurch es auf körperlicher Seite zu Abheilungen etwa von Geschwüren, einer verbesserten arteriellen Durchblutung sowie in seelischer Hinsicht zu mehr Selbstbewusstsein und Eigeninitiative kommt. Anhand von zwei Praxisfällen möchte ich das praktische Vorgehen veranschaulichen.

\section{Fall 1}

Frau G.M. leidet seit 12 Jahren an beidseitigen, ca. $3-4 \mathrm{~cm}$ grossen, kreisrunden, unangenehm riechenden Ulcera cruris (sog. "Gamaschenulcus») auf dem Boden einer chronisch-venösen Insuffizienz. Die Krankheit scheint genetisch prädisponiert zu sein, weil die Grossmutter zeitlebens die gleiche Krankheit gehabt hat. Trotz des Besuchs mehrerer Fachkliniken und der intensiven Therapie durch Spezialisten konnte das Ulcus bisher nicht zum Abheilen gebracht werden. Nachdem die Patientin jede Hoffnung auf konventionelle Hilfe aufgegeben hat, will sie es noch einmal mit Alternativmethoden probieren und kommt deshalb zur Energietestung.

Es findet sich ein Konflikt mit dem Thema «hilflos» im ersten Chakra. Energetisch gehören die Beine erfahrungsgemäss zum ersten Chakra und passen daher aus dieser Betrachtungsweise genau zum geklagten medizini-

\section{KARGER}

Fax +4976145207 14 (c) 2013 S. Karger GmbH, Freiburg
Dr. med. Reimar Banis

c/o Internationale Gesellschaft für Psychosomatische Energetik (IGPSE)

Dörflistrasse 4, 6056 Kägiswil, Schweiz

www.igpse.ch 


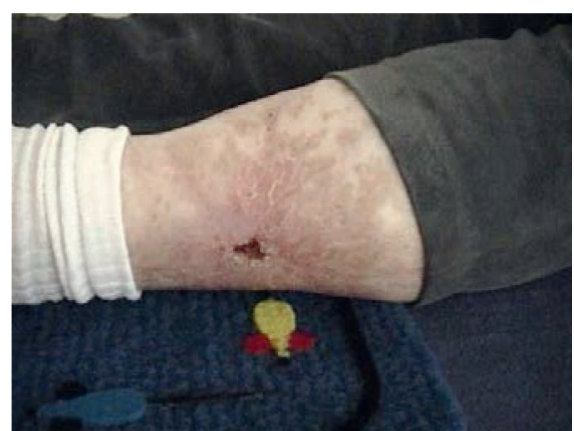

Abb. 2. Abheilendes Ulcus.

schen Problem. Das Konfliktthema ergibt für die Patientin auf Anhieb Sinn, da es sich als unbewältigtes seelisches Thema wie ein roter Faden durch ihr Leben zieht, denn sie hat sich stets hilflos gefühlt. Nach 6-wöchiger Einnahme der EmotionalTropfen (Emvita ${ }^{\circledR}$ 3) ist das Ulcus ohne weiteres Zutun weitgehend abgeheilt (Abb. 2). Das Ulcus flackert später noch zweimal auf - zuerst bei der Testung eines Zahnherdes, der sich als wurzelbehandelter Zahn mit Restostitis entpuppt, und danach bei einem weiteren Konflikt. Nach der Sanierung des Zahnherdes und Abheilung des zweiten Konfliktes schliesst sich auch das neu entstandene, nunmehr nur noch pfenniggrosse Ulcus spontan wieder und bleibt auch dauerhaft geschlossen (Nachbeobachtungszeit über ein Jahr).

\section{Fall 2}

Ein 59-jähriger Mann mit erheblichem Übergewicht (115 kg), starker Raucher, leidet seit 5 Jahren an erheblichen Beinschmerzen und kann nur noch 50 Meter weit gehen. Er leidet allgemein an schneller Ermüdung. Bei der Untersuchung sind die Fusspulse nicht tastbar und die Füsse sind kalt und weisslich marmoriert. Der hinzugezogene Gefässspezialist hat eine Gefässoperation und notfalls eine Beinamputation der fortgeschrittenen pAVK vorgeschlagen. Um alles $\mathrm{zu}$ versuchen, kommt er zur naturheilkundlichen Behandlung, will aber an seinem ungesunden Lebensstil selbst ausdrücklich nichts ändern.
Bei der Energietestung findet sich eine Erdstrahlbelastung, weshalb ein versierter Rutengänger seinen Bettplatz untersucht und das Bett danach auf eine neutrale, ungestörte Zone verschiebt. Als Konflikt testet das Thema «hastig, nervös», was er bestätigen kann, denn als Unternehmer habe er dauernd Stress und käme nie zur Ruhe. Später findet sich bei der zweiten Konsultation ein weiterer Konflikt mit dem Thema unterdrückte Aggressionen (Emvita ${ }^{\circledR} 9$ «Wut»), was er ebenfalls als zutreffend bezeichnet, denn er würde seinen Ärger oft nicht zeigen und sich stattdessen mit gutem Essen, Alkohol und Zigaretten trösten. Nach insgesamt 8-monatiger Therapie ist seine Gehstrecke wieder normal und er kann beim Golfspielen unbegrenzt laufen. Die Füsse sind wieder warm und gut durchblutet. Von sich aus hört er nach der PSE-Therapie erstmals mit dem Rauchen auf, weil er nun einsieht, dass dies für seine Durchblutung nicht gut ist.

Da sich vergleichbare Erfolge bei der Anwendung der PSE bei verschiedenen Therapeuten gezeigt haben [2], kann man davon ausgehen, dass dabei bestimmte gesetzmässige Vorgänge ablaufen, die für den Erfolg verantwortlich sind. Was dabei im Einzelnen abläuft, ist noch unerforscht. Aus der Praxiserfahrung kann man folgern, dass venöse ebenso wie arterielle Durchblutung und das feinstoffliche Energiesystem eng zusammenhängen. Energieblockaden durch seelische Konflikte erzeugen vermutlich einen unterschwelligen Dauerstress des vegetativen Nervensystems, der über segmentale Einflüsse den Blutfluss drosselt. Löst man diese Blockaden in Form unbewusster negativer Emotionen auf, verbessert sich sowohl die allgemeine, unbewusste seelisch-vegetative Daueranspannung des Patienten als auch seine lokale Durchblutungsstörung der Beine.

\section{Fazit}

Als naturheilkundlich tätiger Arzt habe ich vergleichbare spektakuläre Effekte durch andere Verfahren in Jahrzehnten ärztlicher Tätigkeit nie gesehen. $\mathrm{Zu}$ meinem Repertoire gehören die üblichen allgemeinärztlichen Ratschläge, die sattsam bekannt sind, wie Gewichtsabnahme, Aufhören mit dem Rauchen, Bewegungstraining usw., dazu aus naturheilkundlicher Sicht die bewährten OzonEigenblutinfusionen, Schlangengifte (Horvi), Ginkgo bei arteriellen Durchblutungsstörungen sowie Rosskastanie und ähnliche Präparate bei venösen Durchblutungsstörungen. Bei manchen Patienten bewähren sich auch Blutegel; hinzu kommen Milieuverbesserungen durch eine Therapie der häufig gestörten Darmflora, durch Fasten, durch Kneipp usw.

Doch alle üblichen Verfahren erweisen sich oft als wenig wirksam, weshalb effektiv wirksame Alternativen gesucht werden. Mit der PSE scheint eine solche effektive Therapiemethode zur Verfügung zu stehen. Die damit erzielten durchweg guten bis sehr guten Erfolge deuten auf eine enge Beziehung von Durchblutung und feinstofflichem Energiesystem hin. Speziell von Patientinnen hört man immer wieder nach der erfolgreichen PSE-Therapie, sie hätten gegenüber früher deutlich wärmere Hände und Füsse. Die Erfolge selbst bei schwerwiegenden Durchblutungsstörungen zeigen darüber hinaus, dass komplementärmedizinisch mit der PSE stets ein therapeutischer Versuch gewagt werden sollte.

\section{Literatur}

1 Banis R: Heilung durch Energiemedizin. Petersberg, Via Nova, 2012.

2 Holschuh-Lorang B: Psychosomatische Energetik und ihre Auswirkungen auf manifeste Organkrankheiten; in Banis R (Hrsg): Lesebuch der Psychosomatischen Energetik. Sulzbach, Edition Co'med, 2006. 


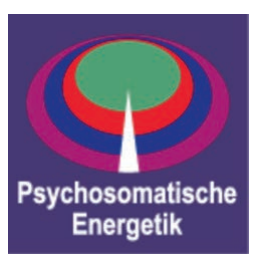

\section{Informationen zur Psychosomatischen Energetik}

Die seit 1997 etablierte Psychosomatische Energetik (PSE) ist dabei, zu einer Standardmethode der modernen Komplementärmedizin zu werden. Sie verbindet uralte Methoden wie den Schamanismus und das indische Yoga mit Psychoanalyse und Homöopathie zu einem neuen Ganzen. Ihre Grundüberlegung geht von der Erfahrung aus, dass ein harmonisches, von seelischen Traumen freies Energiesystem für die seelische und körperliche Gesundheit wichtig ist und eine entsprechende Therapie ganzheitliche Selbstheilprozesse in Gang setzt.

Die Ausbildung zum «zertifizierten Energietherapeut» verläuft berufsbegleitend und setzt sich aus mehreren festgelegten Ausbildungsabschnitten zusammen (Seminardaten siehe www.igpse.ch). Mittlerweile gibt es annähernd 500 ausgebildete Therapeuten im deutschsprachigen Raum. Die PSE wird darüber hinaus auch in Nordamerika sowie weiteren Ländern unterrichtet und mittlerweile in mehr als 20 Ländern weltweit praktiziert.

Infomaterial für Therapeuten mit Hinweisen zur Ausbildung kann bei der Rubimed AG, Grossmatt 3, 6052 Hergiswil/Schweiz (www.rubimed.com) kostenlos angefordert werden. 